Relatório especial

\title{
Doenças infecciosas e parasitárias no Brasil: uma década de transição'
}

\author{
Neir Antunes Paes ${ }^{2}$ e Lenine Angelo A. Silva²
}

RESUMO Em pleno período de transição epidemiológica e demográfica, também marcado pela melhoria na qualidade dos registros de óbitos no Brasil, é crucial entender o comportamento recente da mortalidade por doenças infecciosas e parasitárias neste país. Este trabalho apresenta as mudanças nos padrões de mortalidade por causas infecciosas e parasitárias para o Brasil e seus estados durante a década de 1980. Foram utilizados para tanto os dados de mortalidade provenientes do Sistema de Informações sobre Mortalidade do Ministério da Saúde, classificados de acordo com a Classificação Internacional de Doenças (nona revisão). O resultado desta análise revela mudanças nos padrões de mortalidade com acentuadas quedas nas taxas de mortalidade por doenças infecciosas e parasitárias para o Brasil (variação de $41 \%$ para homens e $44 \%$ para mulheres), em particular para os estados das regiões Norte e Nordeste. No entanto, estes estados ainda são detentores das mais elevadas taxas de mortalidade do país. Em termos de distribuição etária, as variações atingiram com maior intensidade os extremos de idade, especialmente o grupo de menores de 1 ano. Dentre as doenças infecciosas e parasitárias, o estudo observou ainda o comportamento da mortalidade por doenças infecciosas intestinais (na sua maioria classificadas como mal definidas), tuberculose e septicemia. As taxas de mortalidade por doenças infecciosas intestinais e tuberculose apresentaram uma expressiva queda; o risco de morte por septicemia, por sua vez, apresentou um aumento real durante a década. Apesar do decréscimo global das taxas de mortalidade e da diminuição da mortalidade proporcional por doenças infecciosas e parasitárias, a mortalidade por esta causa ainda permanece elevada no Brasil, e exige atenção prioritária por parte dos setores competentes.

Uma das características mais marcantes da modificação do padrão brasileiro de mortalidade nos últimos 20

\footnotetext{
1 A análise desenvolvida no presente artigo é parte do projeto integrado de pesquisa intitulado "O poder explicativo dos registros de óbitos para estimar taxas de mortalidade no Brasil", desenvolvido com o apoio do Conselho Nacional de Desenvolvimento Científico e Tecnológico (CNPq) (processo no. 523.355/94-5)

2 Universidade Federal da Paraíba, Departamento de Estatística. Correspondência e pedidos de separatas devem ser enviados a Neir Antunes Paes no seguinte endereço: Departamento de Estatística, Centro das Ciências Exatas e da Natureza (CCEN), Universidade Federal da Paraíba, Campus - I, CEP 58051-910, João Pessoa, PB, Brasil. Fax: +55-83-2167117; e-mail: antunes@de.ufpb.br
}

anos é o decréscimo das doenças infecciosas e parasitárias (DIP) como causa de morte. O comportamento geral da mortalidade por causas no mundo, em particular da mortalidade decorrente de doenças infecciosas e parasitárias e de doenças crônico-degenerativas, contribuiu para a formulação da teoria da transição epidemiológica, segundo a qual ocorreria uma queda geral na mortalidade com um aumento geral da morbidade. Ao mesmo tempo, o risco de morte por doenças infecciosas e parasitárias diminuiria e perderia participação como foco prioritário de ação, enquanto que ocorreria um aumento da morbidade por doenças crônico- degenerativas, com mudanças no padrão de mortalidade (1). Desde então, essa teoria ganhou força e, a partir dela, surgiram modelos que sustentam e complementam a idéia de transição epidemiológica $(2,3)$.

As DIP têm ocupado um papel relevante entre as causas de morte no Brasil. Este grupo de doenças se reveste de importância por seu expressivo impacto social, já que está diretamente associado à pobreza e à qualidade de vida, enquadrando patologias relacionadas a condições de habitação, alimentação e higiene precárias. Além disso, a análise do comportamento das DIP pode servir para avaliar as condi- 
ções de desenvolvimento de determinada região, através da relação entre níveis de mortalidade e morbidade e condições de vida da população.

Tendo em vista este quadro de transição, assim como a importância das DIP no Brasil, torna-se imprescindível avaliar a mortalidade por doenças infecciosas e parasitárias no país, situando estes dados em um momento histórico também de transição demográfica, que tem concorrido ao envelhecimento da população.

Sabe-se que no Brasil, assim como em outras regiões em desenvolvimento, o registro dos óbitos é problemático. A capacidade diagnóstica dos serviços de saúde e a produção de dados estatísticos confiáveis podem encontrar-se comprometidas por questões diversas, por exemplo de cunho social e político-administrativo. Este comprometimento se reflete principalmente no elevado número de óbitos registrados sem uma causa bem definida e na subenumeração de causas de óbitos perfeitamente notificáveis, problema este ainda persistente no Brasil. Os óbitos por doenças infecciosas e parasitárias, em particular, parecem estar mais sujeitos aos fatores que levam à má notificação. As DIP atingem uma expressiva população menos privilegiada, de baixa renda, com baixo nível escolar e que não dispõe de condições de saneamento básico e assistência primária à saúde, sendo, portanto, mais susceptível à não notificação dos óbitos.

Apesar desses problemas, tem sido sensível a melhora do sistema de registro, compilação e divulgação dos dados a respeito dos eventos vitais e, apesar da insuficiência do sistema de atenção à saúde ser realidade brasileira, a capacidade diagnóstica também tem aumentado. Os programas instituídos pelo governo (com destaque para a criação do sistema de vigilância epidemiológica), a criação de um sistema de notificação compulsória de doenças, com padronização dos formulários de registros de enfermidades, e o interesse do Ministério da Saúde em coletar, centralizar e cuidar da divulgação das informações sobre morbidade e mortalidade são exemplos dessa melhoria.

Estimativas feitas por Paes (4) dão conta de que, em 1980, cerca de $85 \%$ dos óbitos foram registrados no país e, em 1990, 87\% (5). Admitindo-se a limitação dos dados, autores como Paes (4, 5), Chackiel (6) e Preston (7) conferem representatividade suficiente para que tais níveis de cobertura de óbitos permitam que se trace satisfatoriamente um perfil de mortalidade expresso em níveis, padrões e tendências no Brasil. Apesar das restrições, adota-se aqui o pressuposto de que essas colocações são válidas para o subconjunto de óbitos por doenças infecciosas e parasitárias no Brasil.

Assim, o objetivo do presente estudo é traçar o perfil da mortalidade por doenças infecciosas e parasitárias no Brasil e nos seus estados, por sexo e grupos etários, na década de 1980. Este período foi selecionado porque, além de se constituir no primeiro período de consolidação oficial sistemática das declarações de óbitos no país, uniformizada para todos os estados brasileiros, coincide com importante momento, em que se processaram profundas transformações no perfil de mortalidade brasileira. A análise destacou ainda o perfil de mortalidade por doenças infecciosas intestinais, tuberculose e septicemia, subgrupos que compõem o grupo das doenças infecciosas e parasitárias, por sua importância histórica, magnitude e comportamentos particularizados.

\section{METODOLOGIA}

Foi construída uma base de dados a partir das declarações de óbito registradas pelo Ministério da Saúde durante o período de 1979 a 1993 e que se encontram disponíveis através do Sistema de Informação sobre Mortalidade (SIM) (8), onde os óbitos são categorizados de acordo com a Classificação Internacional de Doenças em sua nona revisão (CID-9). As causas de morte são agrupadas em 17 grandes capítulos e as doenças infecciosas e parasitárias estão representadas no pri- meiro capítulo (I). A Classificação Brasileira (CID-Br) também é adotada.

Para o presente estudo, procurou-se descrever a mortalidade por sexo e grupos etários para o Brasil e estados com base na média dos triênios de 1979 a 1981 e 1984 a 1986, assim como para o biênio 1989 e 1990, buscando, deste modo, minimizar o efeito de eventuais flutuações aleatórias (por exemplo, os dados para 1991 não estavam disponíveis para todas as unidades da federação no momento da realização deste trabalho). Por sua vez, as populações utilizadas como denominador para o cálculo das taxas de mortalidade foram extraídas dos censos demográficos de 1980 e 1991, produzidos pela Fundação Instituto Brasileiro de Geografia e Estatística (IBGE) $(9,10)$.

Como o estado de Tocantins foi oficialmente criado em 1988, a partir de um desmembramento do Estado de Goiás, considerou-se os dois estados em conjunto para todo o período analisado. Dessa forma, os dados listados para Goiás englobam também as informações relativas a Tocantins.

Para avaliação do comportamento da mortalidade por doenças infecciosas e parasitárias, foram calculados, para ambos os sexos, a mortalidade proporcional, taxas de mortalidade padronizadas para grandes grupos (a padronização foi feita tomando-se como referência a estrutura etária da população do Brasil em 1990) e anos potenciais de vida perdidos (APVP) (11). Os APVP foram calculados utilizando-se a seguinte fórmula:

$$
A P V P=\frac{\sum_{i=1}^{I} N_{i} \frac{r_{i}}{n_{i}} \cdot F_{i}}{N}
$$

onde $n_{i}$ é a população padrão na classe $i ; r_{i}$ é o número de óbitos na população estudada, na classe $i$; $N_{i}$ é a população estudada, na classe $i$; $N$ é o total da população estudada; e $F_{i}$ é a diferença entre uma idade limite $(E)$ estabelecida (neste caso, 75 anos) e o valor médio $\left(I_{i}\right)$ das idades que integram cada grupo etário. 


\section{RESULTADOS E DISCUSSÃO}

A tabela 1 mostra o comportamento da mortalidade proporcional por DIP em relação aos demais grupos de causas de óbitos. A comparação entre estes dados revela um decréscimo na importância das DIP ao longo da década de 80, tanto para o sexo masculino quanto para o feminino. No entanto, a mortalidade por DIP permanece elevada, o que denuncia a persistência dos problemas que resultam nos óbitos por essa causa. No mesmo período, observa-se que o risco de morrer (ou a taxa de mortalidade) por DIP também diminuiu, permanecendo mais elevado para o sexo masculino.

Em 1980, as taxas de mortalidade por doenças infecciosas e parasitárias giravam em torno de 6,1 óbitos por 10000 homens e 4,3 por 10000 mulheres. Em 1990, essas taxas continuavam mais elevadas para os homens (3,6 óbitos por 10000 homens) do que para as mulheres (2,4 óbitos por $10000 \mathrm{mu}$ lheres); mesmo assim, decresceram de forma considerável (decréscimo de $41 \%$ para homens e $44 \%$ para mulheres). Em 1980, as DIP ocupavam o quarto e o terceiro lugar entre as causas de morte dos sexos masculino e feminino, respectivamente. Em 1990, o qua- dro hierárquico alterou-se e as doenças infecciosas perderam uma posição em ambos os sexos.

Entre as causas de óbitos conhecidas, em 1980, as doenças infecciosas e parasitárias ficaram entre as duas primeiras em quantidade de anos potenciais de vida perdidos. Para o sexo masculino, o número de anos potenciais de vida perdidos por DIP (354,7 anos por 10000 homens) ficou em segundo lugar e só foi menor do que os APVP por afecções perinatais. Para o sexo feminino, no mesmo ano, as DIP foram a maior causa de anos potenciais de vida perdidos (269,7 anos por 10000 mulheres).

Em 1990, o risco de morte por doenças infecciosas e parasitárias caiu de forma considerável para ambos os sexos, ainda permanecendo mais elevado para os homens do que para as mulheres. Para o sexo masculino, perdeu-se cerca de 142,6 anos por 10000 homens. No sexo feminino, esses valores ficaram em torno de 99,1 anos por 10000 mulheres. Esses valores ainda parecem elevados; o prejuízo nacional provocado pelas doenças infecciosas e parasitárias em anos potenciais perdidos é extremamente elevado e assume maior importância quando fica comprovado que se está morrendo de uma causa que, diferentemente das demais, é de fácil prevenção, denotando que os avanços obtidos no âmbito social e da saúde durante a década foram insuficientes para manter o controle das doenças infecciosas e parasitárias no Brasil.

Quando se analisa o perfil de óbitos da população por doenças infecciosas e parasitárias em termos de faixa etária, evidencia-se que o maior número de óbitos ocorreu entre a população menor de 1 ano e o grupo de 1 a 4 anos, em ambos os sexos. Esta evidência pode ser justificada pelo fato de esses dois grupos etários, em particular o primeiro (grupo base para o estudo da mortalidade infantil), serem altamente susceptíveis ao meio externo, o que permite concluir que quanto menor a atenção dispensada a esses grupos, maior será a mortalidade nessa faixa etária.

No período analisado, a mortalidade proporcional causada por DIP cai rapidamente à medida em que a idade aumenta até a faixa dos 15 aos 19 anos, para ambos os sexos, em particular para o sexo masculino. A partir daí o padrão etário se diferencia. Para o sexo masculino, segue-se um leve aumento até o grupo etário dos 35 aos 39 anos e uma redução lenta mas consistente até 70 anos e mais. Para o sexo

TABELA 1. Mortalidade proporcional por sexo segundo grandes grupos de causas, taxas padronizadas de mortalidade e anos potenciais de vida perdidos, Brasil, 1980 a 1990

\begin{tabular}{|c|c|c|c|c|c|c|c|c|c|c|c|c|c|c|}
\hline \multirow[b]{3}{*}{ Grupos de causas } & \multicolumn{6}{|c|}{ Mortalidade proporcional } & \multicolumn{4}{|c|}{ Taxas padronizadas de morte ${ }^{a}$} & \multicolumn{4}{|c|}{$\begin{array}{c}\text { Taxas de anos potenciais } \\
\text { de vida perdidos }{ }^{a}\end{array}$} \\
\hline & \multicolumn{3}{|c|}{ Masculino } & \multicolumn{3}{|c|}{ Feminino } & \multicolumn{2}{|c|}{ Masculino } & \multicolumn{2}{|c|}{ Feminino } & \multicolumn{2}{|c|}{ Masculino } & \multicolumn{2}{|c|}{ Feminino } \\
\hline & 1980 & 1985 & 1990 & 1980 & 1985 & 1990 & 1980 & 1990 & 1980 & 1990 & 1980 & 1990 & 1980 & 1990 \\
\hline \multicolumn{15}{|l|}{ Doenças infecciosas e } \\
\hline parasitárias & 9,34 & 6,64 & 5,32 & 9,37 & 6,86 & 5,29 & 6,06 & 3,58 & 4,32 & 2,41 & 354,7 & 142,6 & 269,7 & 99,1 \\
\hline Neoplasmas & 7,87 & 8,36 & 9,26 & 8,81 & 9,73 & 11,28 & 6,35 & 6,24 & 5,32 & 5,13 & 70,5 & 68,4 & 62,9 & 63,2 \\
\hline \multicolumn{15}{|l|}{ Doenças do aparelho } \\
\hline circulatório & 23,58 & 24,53 & 25,62 & 27,81 & 26,65 & 31,92 & 19,44 & 17,25 & 17,51 & 14,53 & 160,9 & 150,5 & 114,3 & 98,8 \\
\hline \multicolumn{15}{|l|}{ Doenças do aparelho } \\
\hline respiratório & 7,85 & 7,85 & 8,23 & 8,08 & 8,25 & 8,65 & 5,59 & 5,54 & 4,20 & 3,94 & 212,2 & 119,9 & 165,0 & 85,3 \\
\hline Afecções perinatais & 7,19 & 5,85 & 4,75 & 7,11 & 5,99 & 5,06 & 4,09 & 3,20 & 2,89 & 2,30 & 359,8 & 223,7 & 256,7 & 161,2 \\
\hline Causas mal definidas & 20,09 & 20,55 & 17,45 & 21,78 & 2,25 & 19,33 & 14,79 & 11,45 & 11,90 & 8,80 & 467,7 & 242,8 & 362,2 & 165,2 \\
\hline Causas externas & 9,19 & 11,52 & 2,31 & 2,37 & 9,67 & 11,52 & 17,10 & 2,37 & 4,89 & 15,10 & 336,3 & 424,8 & 85,9 & 82,6 \\
\hline Demais causas & 8,03 & 8,27 & 6,48 & 6,04 & 8,20 & 8,27 & 12,28 & 6,04 & 12,38 & 11,12 & 276,0 & 212,1 & 219,4 & 140,3 \\
\hline
\end{tabular}

Fonte: Brasil, Ministério da Saúde, Sistema de Informações de Mortalidade, CD-ROM 1979 a 1993.

a Por 10000 habitantes. 
feminino, a partir do grupo etário dos 20 aos 24 anos, segue-se um decréscimo contínuo do percentual de óbitos por DIP até os 70 anos e mais.

A redução da proporcionalidade das doenças infecciosas e parasitárias como causa de morte, observada nos grupos etários mais avançados, se justifica em parte pelo aumento proporcional das mortes por doenças crônicodegenerativas. É claro o descenso na importância das DIP ocorrido na década para todas as faixas etárias, sendo mais forte no primeiro quinquênio.

Especificamente dentre as doenças infecciosas e parasitárias, destaca-se o perfil de mortalidade por doenças infecciosas intestinais, tuberculose e septicemia. O gráfico 1 mostra a contribuição destes três grupos para a mortalidade por DIP em ambos os sexos. A seguir, discute-se a contribuição de cada uma destas subcausas para as taxas de mortalidade por DIP.

\section{Doenças infecciosas intestinais}

Das três subcausas selecionadas, as doenças infecciosas intestinais abarcam a maior fatia das DIP como causa de óbitos. Praticamente 90\% das doenças infecciosas intestinais são caracterizadas como mal definidas (desconhece-se o real agente etiológico causador da infecção). Em última análise, isto dificulta a produção de estatísticas de maior poder preditivo.

É importante ressaltar que a morte por doenças infecciosas intestinais denuncia problemas com hábitos alimentares, saneamento básico e tratamento da água, dentre outros, e, no outro extremo, com a capacidade de defesa do organismo do paciente enfermo, em particular da criança (no Brasil, o grupo mais acometido pelas doenças infecciosas intestinais). Aqui a desnutrição repercute nos indivíduos menos favorecidos, os quais se tornam susceptíveis aos agentes desencadeadores dessas enfermidades, resultando na morte por uma causa certamente evitável. As elevadas taxas de mortalidade por doenças infecciosas intestinais em 1980 se devem principalmente ao elevado número de óbitos no grupo de crianças

GRÁFICO 1. Mortalidade proporcional pelas principais causas de morte no grupo das doenças infecciosas e parasitárias, Brasil, 1980 a 1990

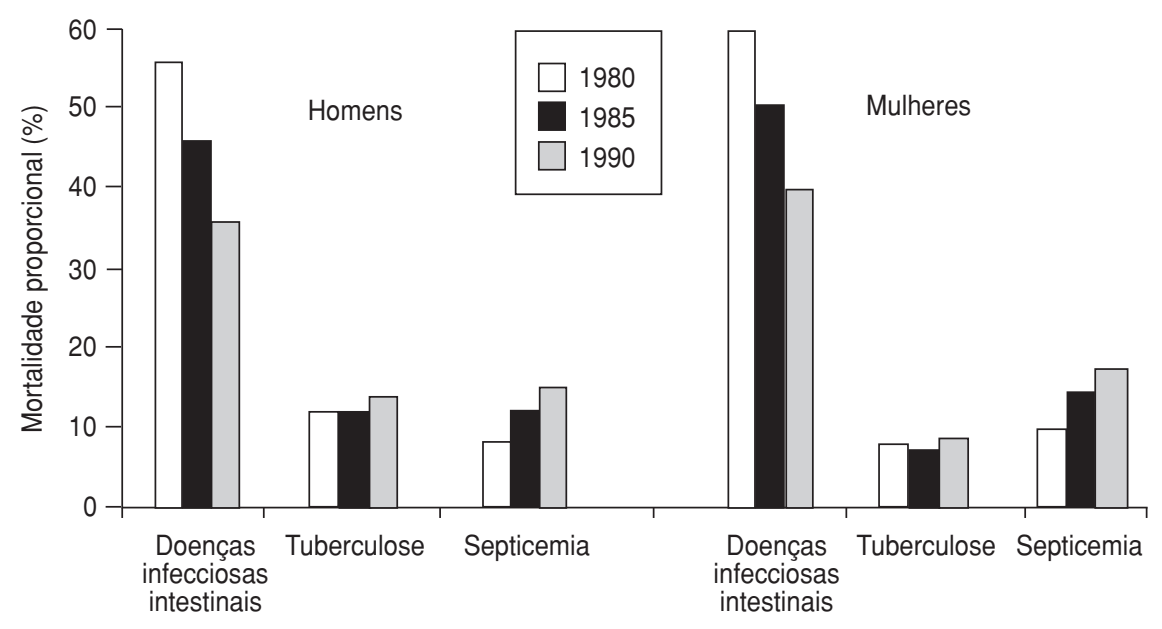

Fonte: Brasil, Ministério da Saúde, Sistema de Informações de Mortalidade, CD-ROM 1979-1993.

menores de 1 ano, assim como a queda na taxa de mortalidade infantil por doenças infecciosas intestinais em 1990 também resultou na diminuição do risco de morte geral da população por doenças infecciosas e parasitárias.

A mortalidade proporcional por doenças infecciosas intestinais por grupos etários se comportou de forma muito semelhante em ambos os sexos e sem grande variação ao longo da década (gráfico 2). O grupo menor de 1 ano respondeu pela grande maioria dos óbitos dessa subcausa e, em seguida, houve uma brusca queda na mortalidade proporcional até o grupo dos 15 aos 19 anos, mantendo-se praticamente constante, a partir de então, até os 64 anos. Em ambos os sexos houve uma discreta diminuição dos percentuais ao longo da década.

\section{Tuberculose}

As doenças infecciosas intestinais e a septicemia são causas que apresentam como população alvo os extremos etários, em particular a população de menores de 1 ano. A tuberculose, ao contrário, é uma endemia de elevada morbidade e que manifesta elevação dos riscos de morte na população adulta, seqüela de lesões que levaram anos para evoluir, muitas vezes de forma subclínica. Tem-se discutido o aumento da morbi-mortalidade por tuberculose, associado ao crescente número de casos de tuberculose em pacientes aidéticos na década de 1990, além da já sabida associação com os efeitos deletérios do fumo. Esses fatores, associados às campanhas de controle e prevenção à tuberculose e, em menor grau, às campanhas de combate ao fumo e à AIDS, têm contribuído para alterar o perfil da evolução natural da doença (12).

Questão de saúde pública, o controle da tuberculose também sofre os efeitos das políticas de administração e liberação de recursos para o combate à doença. A década de 1980, em especial o primeiro qüinqüênio, foi marcada pela queda nas taxas de mortalidade por tuberculose, o que não necessariamente significa controle da doença. Sabe-se que a queda nas taxas de mortalidade não foi acompanhada pela diminuição da incidência e prevalência dessa doença, o que torna a análise dos indicadores de mortalidade pouco preditiva quanto à sua evolução, surgindo assim a necessidade de análise de indicadores 
GRÁFICO 2. Mortalidade proporcional por doenças infecciosas intestinais, por grupos etários e sexo, Brasil, 1980 a 1990
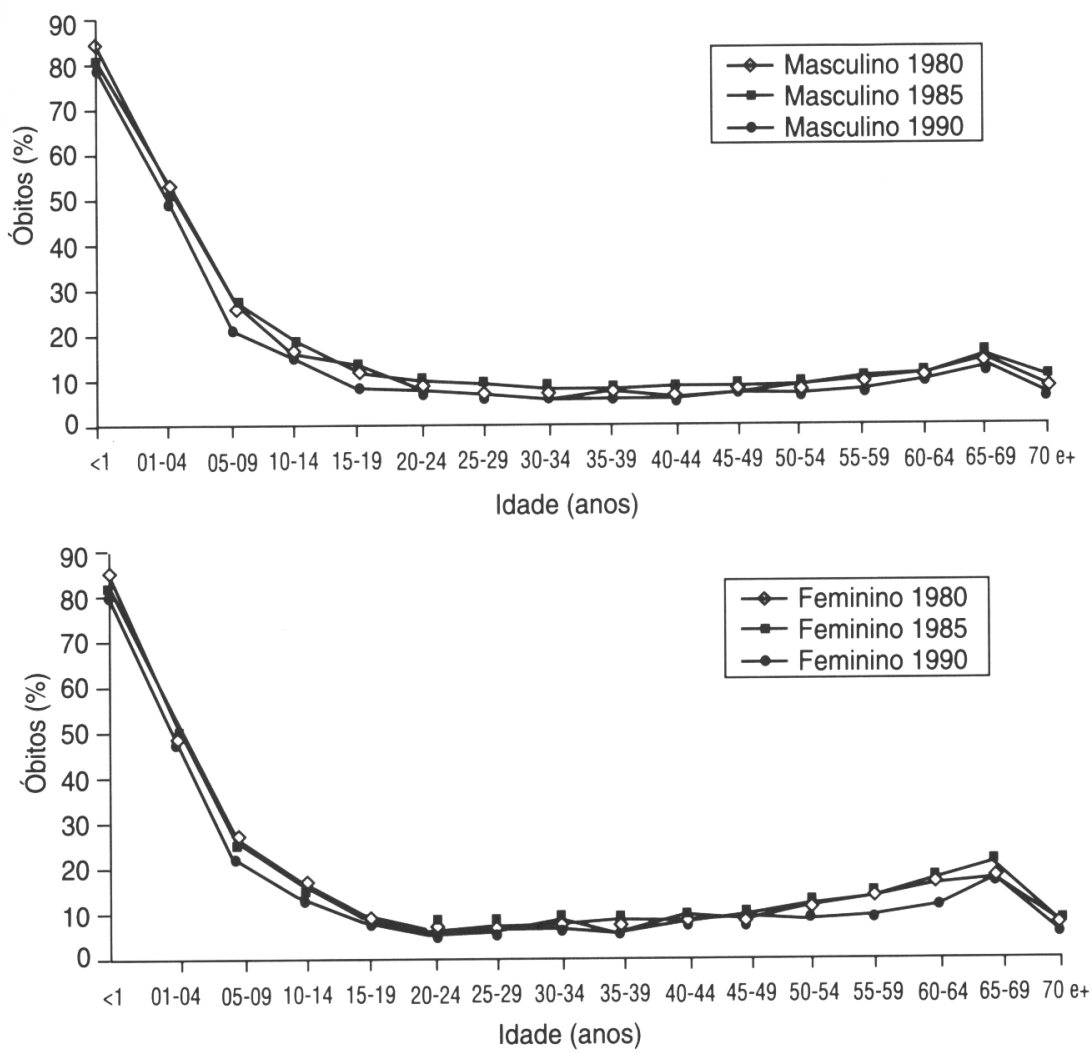

Fonte: Brasil, Ministério da Saúde, Sistema de Informações de Mortalidade, CD-ROM 1979-1993.

de morbidade, os quais não fazem parte dos objetivos deste trabalho.

O comportamento da mortalidade proporcional por tuberculose por grupos etários na década de 80 foi bastante semelhante para ambos os sexos (gráfico 3). Diferentemente das doenças infecciosas intestinais, a tuberculose não apresentou importância significativa na mortalidade proporcional do grupo de menores de 1 ano, tendo predominado nos grupos etários da idade adulta e senil. Para o sexo masculino, sua contribuição como causa de morte aumentou gradativamente atingindo valor máximo na faixa dos 35 aos 39 anos e decrescendo então até 70 anos e mais. É importante verificar que até o grupo dos 40 aos 44 anos observase uma redução na mortalidade proporcional por grupos etários de 1980 para 1985, sendo que, no qüinqüênio seguinte, houve um aumento a partir deste grupo etário.

Para o sexo feminino, o valor mais elevado ocorreu no grupo etário dos 20 aos 24 anos, apresentando decréscimo até 70 anos e mais, sendo verificados alguns grupos etários que não seguiram um padrão de decréscimo regular. Persiste aqui a diferença no comportamento dos óbitos ao longo da década, sendo que a tendência de decréscimo só se acentuou a partir de 40 a 44 anos. De forma semelhante ao ocorrido para o sexo masculino, para o sexo feminino, em praticamente todos os grupos etários até a faixa dos 50 aos 54 anos, a mortalidade proporcional aumentou na segunda metade da década.

Verificou-se uma diminuição no risco de morte para ambos os sexos na década. Os maiores riscos ocorreram para o sexo masculino, sendo o comportamento por grupos etários bastante particular.

\section{Septicemia}

Sabe-se, quanto ao risco de morte, que a septicemia representa a fase evo- lutiva final de um processo infeccioso que se instala no organismo e que existem diversos fatores que, relacionados ou não ao organismo (capacidade de resposta imune do paciente, virulência do agente, poder de eficácia da terapêutica utilizada), decidirão pelo êxito ou pelo óbito do paciente. Assim sendo, a septicemia estaria relacionada aos fatores predisponentes à infecção, atingindo grupos etários mais frágeis do ponto de vista imunológico.

Já se pode presumir que a septicemia apresenta características epidemiológicas semelhantes às doenças infecciosas intestinais e à tuberculose, com o detalhe de que esta enfermidade só pode ser diagnosticada por um médico, o que condiciona seu diagnóstico à disponibilidade de assistência médica. Este fato resulta em um elevado nível de sub-registro e também em um desvio desses óbitos para outros grupos de causas, em especial para as causas de morte mal definidas, tendo em vista a baixa cobertura da assistência médica verificada no Brasil (5). O uso indiscriminado da antibioticoterapia, a epidemia de AIDS, pobreza e fome, a incapacidade dos serviços de saúde de apresentarem um atendimento adequado e de acompanharem os pacientes são fatores fortemente relacionados ao aumento do número de óbitos por septicemia.

A septicemia apresentou um comportamento também particular (gráfico 4). Para o sexo masculino, a mortalidade proporcional por grupos etários começou a aumentar a partir do grupo de menores de 1 ano até a faixa dos 10 a 14 anos, havendo declínio até a faixa dos 40 a 44 anos, voltando a subir dos 65 aos 69 anos e decrescendo em seguida até o grupo com 70 anos ou mais. Percebe-se, assim, que a população adulta foi menos acometida por septicemia, mas houve aumento da mortalidade proporcional para todos os grupos etários ao longo da década.

A mesma irregularidade de comportamento foi verificada para o sexo feminino, sendo seus níveis mais elevados. Houve diferenças também na participação percentual dos grupos etários nos diferentes anos. Na tentativa de se estabelecer um perfil, po- 
GRÁFICO 3. Mortalidade proporcional por tuberculose, por grupos etários e sexo, Brasil, 1980 a 1990
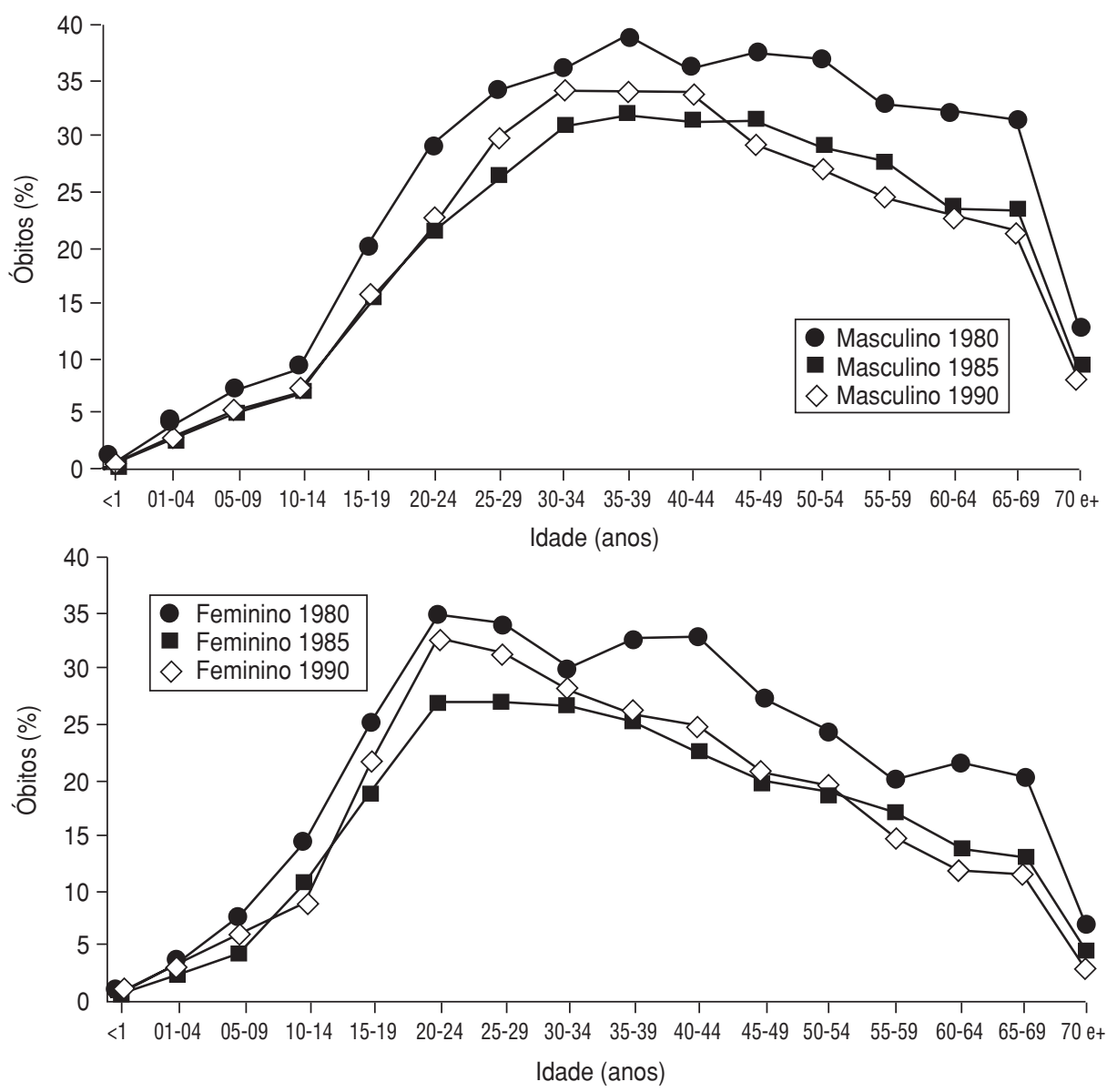

Fonte: Brasil, Ministério da Saúde, Sistema de Informações de Mortalidade, CD-ROM 1979-1993.

dese observar que os valores ascendem a partir do grupo menor de 1 ano até o grupo dos 15 aos 19 anos, decrescendo até a faixa de 35 a 39 anos, voltando a subir até 69 anos e caindo novamente em maiores de 70 anos. Dentre as DIP, a septicemia constituiu a única causa em que houve real aumento de risco de morte ao longo da década, para ambos os sexos.

\section{As doenças infecciosas e parasitárias nos estados brasileiros}

Sabe-se que os estados brasileiros apresentam graus diferentes de desenvolvimento, que se refletem no nível de vida da população e repercutem no padrão de mortalidade por causas específicas. A tabela 2 apresenta as taxas gerais de mortalidade por DIP para ambos os sexos e a variação percentual de 1980 a 1990. O que se observa é que o risco de morte por doenças infecciosas e parasitárias diminuiu de forma considerável.

Em 1980, para o sexo masculino, a variação das taxas para todos os estados oscilou entre 2,6 e 9,5 em 10000 homens, excetuando-se o estado de Rondônia, com 13,05 em 10000 homens. Já em 1990 essa variação foi de 2,1 a 7,4 para cada 10000 homens. Para o sexo feminino, em 1980, o risco de morte por DIP variou de 2,0 a 7,5 óbitos em 10000 mulheres, excetuando-se Rondônia, com 10,70 óbitos em 10000 mulheres. Em 1990, a variação do risco de morte foi de 1,2 a 4,4 óbitos para cada 10000 mulheres, excetuando-se Rondônia e Roraima, com 5,94 e 6,13 óbitos em 10000 mulheres, respectivamente.
Em 1980, os estados das regiões Norte e Nordeste detinham as mais altas taxas de mortalidade. Nesse mesmo ano, para o sexo masculino, somente 12 dos 26 estados brasileiros apresentavam taxas de mortalidade mais baixas que a média nacional, que era de 6,06 óbitos por 10000 homens. Rondônia foi o estado detentor da mais alta taxa de mortalidade por DIP, enquanto que o Maranhão apresentou o menor risco de morte pela mesma causa (2,55 óbitos/10 000 homens). Dentre as regiões mais desenvolvidas, destaca-se a elevada taxa de mortalidade masculina do Distrito Federal (8,00 óbitos/10 000 homens).

Em 1990, todos os estados apresentaram queda nas taxas de mortalidade por DIP. A única exceção ocorreu para a população masculina do estado do Mato Grosso, que teve o risco de morte aumentado de 2,93 para 3,41 óbitos (16\%). As maiores quedas nas taxas de mortalidade entre os homens ficaram por conta dos estados do Rio Grande do Sul, Santa Catarina, Paraná, São Paulo, Espírito Santo, Minas Gerais, Alagoas, Paraíba, Rio Grande do Norte, Ceará, Pará e Amazonas, todos com quedas relativas maiores que $40 \%$ e superiores à variação nacional, sendo que o Rio Grande do Sul, em 1990, foi o estado detentor das menores taxas de mortalidade por doenças infecciosas e parasitárias para o sexo masculino $(2,05$ óbitos /10 000 homens).

Quanto ao sexo feminino, o maior risco de morte, em 1980, pertenceu ao estado de Rondônia (10,70 óbitos/ 10000 mulheres), enquanto que as menores taxas de mortalidade (assim como para o sexo masculino) foram registradas no Maranhão (2,01 óbitos/ 10000 mulheres).

Vale a pena ressaltar a limitação na qualidade dos dados dos registros de óbitos para alguns estados. Dos estados da região Nordeste, o Piauí, por exemplo, apresentou o menor risco de morte por doenças infecciosas e parasitárias para os homens em 1980, enquanto que seu sub-registro de óbitos foi da ordem de $46 \%$, com $40 \%$ de causas mal definidas (5). Situação oposta foi observada para a população mas- 
GRÁFICO 4. Mortalidade proporcional por septicemia, Brasil, por grupos etários e sexo, 1980 a 1990
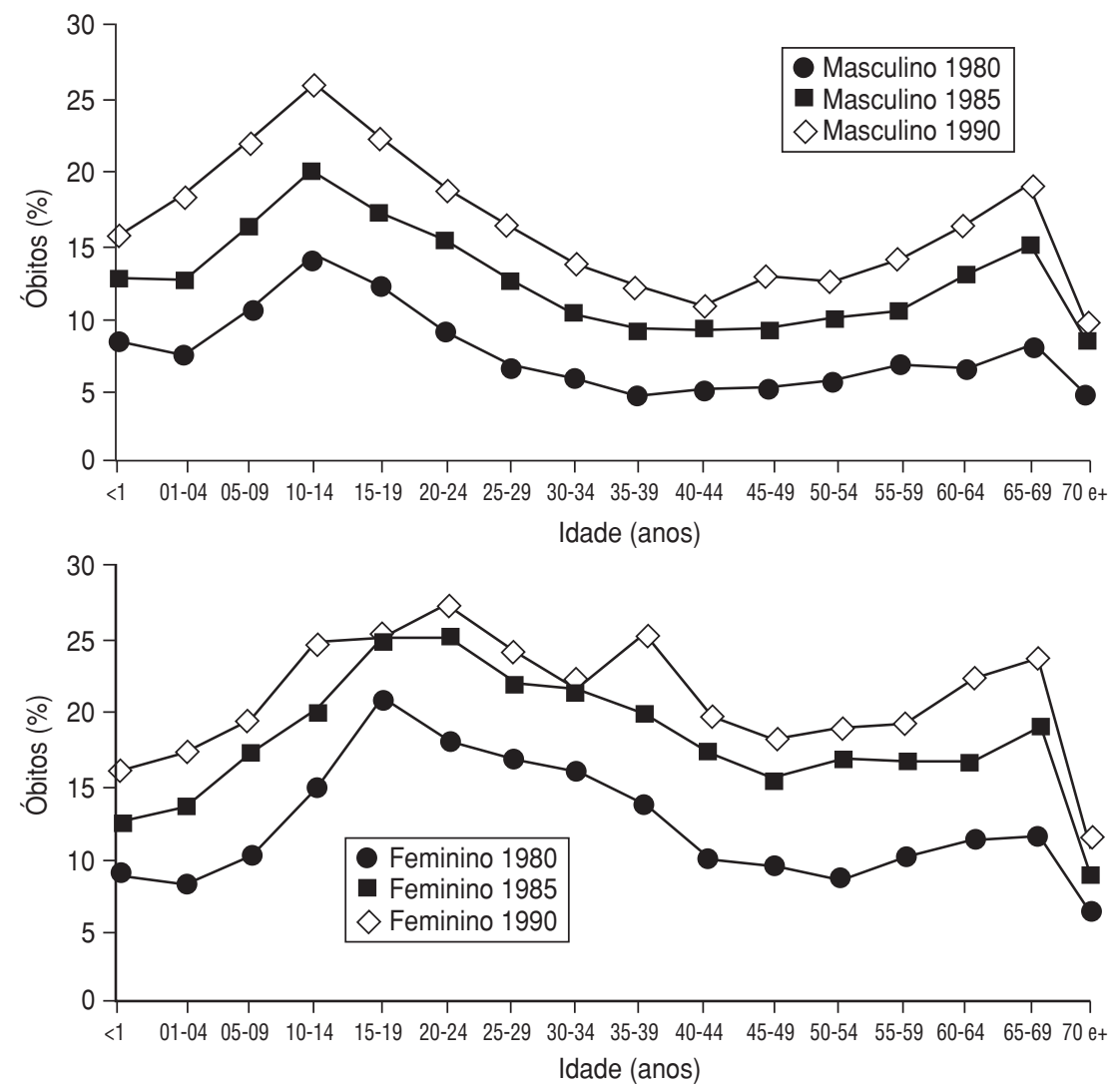

Fonte: Brasil, Ministério da Saúde, Sistema de Informações de Mortalidade, CD-ROM 1979-1993.

culina do Distrito Federal, cujo subregistro, para o mesmo ano, foi da ordem de $4 \%$, com $1,5 \%$ de causas mal definidas. É possível especular que os estados com elevado sub-registro de óbitos e com elevado percentual de causas mal definidas (quase todos os estados do Norte e Nordeste) tenham as taxas de mortalidade por doenças infecciosas e parasitárias mais elevadas que as apresentadas aqui. Portanto, na comparação entre os estados, é ao perfil hierárquico dos níveis de mortalidade que mais se deve dar atenção, e não à magnitude dos níveis expressos pelas taxas.

\section{Participação das subcausas na mortalidade por estado}

A tabela 3 apresenta as taxas de mortalidade por doenças infecciosas intestinais, tuberculose e septicemia, assim como sua variação em percentuais durante a década de 80 , para ambos os sexos, no Brasil e estados.

Todos os estados apresentaram variações negativas nas taxas de mortalidade por doenças infecciosas intestinais, sendo que as maiores variações foram de $66 \%$ para o sexo masculino no Rio Grande do Sul (de 1,36 para 0,46 óbitos/10 000 homens) e 65\% para o sexo feminino no estado de São Paulo (queda de 2,20 para 0,76 óbitos/10 000 mulheres). As menores variações ocorreram em Pernambuco (queda de $22 \%$, sexo masculino) e Amapá (queda de $14 \%$, sexo feminino). Em 1980, dos 12 estados com taxas superiores à nacional (2,99 óbitos / 1000 homens) para o sexo masculino, 10 se situavam no Norte e no Nordeste do país. Para 1990, os menores riscos de morte ficaram para os estados do Maranhão, Piauí, Santa Catarina, Rio Grande do Sul e São Paulo, os quais apresentaram taxas inferiores a 1 óbito para cada 10000 homens.

O comportamento da mortalidade por doenças infecciosas intestinais difere pouco para as mulheres, sendo que, no início da década, somente os estados do Amapá, Maranhão, Piauí, Ceará, Espírito Santo, São Paulo, Paraná, Santa Catarina e Rio Grande do Sul apresentavam taxas de mortalidade menores que a média nacional, passando os demais estados a se posicionarem no lado oposto. As mais altas taxas de mortalidade para o sexo feminino, no início da década, ficaram para os estados de Amazonas, Roraima e Alagoas, todos com riscos de morte superiores a 4 óbitos/10 000 mulheres, sendo que as quedas nas taxas de mortalidade para o sexo feminino se deram com similaridade ao ocorrido para o sexo masculino.

A tendência do risco de morte por tuberculose foi de queda na década de 1980. A exceção se deu para o sexo masculino no estado do Piauí, cujas taxas de mortalidade se mantiveram constantes, e para o sexo feminino no Acre e Roraima, cujo risco de morte aumentou, resultado talvez de uma melhoria na capacidade diagnóstica (tabela 3). O risco de morte por tuberculose foi maior para o sexo masculino que para o sexo feminino e esse comportamento se repetiu durante toda a década, sendo que, para a maioria dos estados, a queda nas taxas de mortalidade por tuberculose também ocorreu em maior magnitude para as mulheres que para os homens.

As taxas de mortalidade para o sexo masculino no final da década de 80 foram, em sua maioria, mais elevadas do que para o sexo feminino no início da década. Sugere-se, assim, que as taxas de mortalidade por tuberculose para os homens refletem um atraso de pelo menos 10 anos em comparação aos níveis alcançados para o sexo feminino. Para o sexo masculino, os estados do Maranhão, Piauí, Ceará, Rio Grande do Norte, Paraíba, Alagoas, Sergipe, São Paulo, Paraná, Santa Catarina, Mato Grosso, Goiás e Distrito Federal apresentaram riscos de morte inferiores à média nacional no início da década (0,78 óbitos/10 000 homens). 
TABELA 2. Taxas padronizadas de mortalidade por doenças infecciosas e parasitárias conforme sexo e variação percentual na década, Brasil, 1980 a $1990^{a}$

\begin{tabular}{|c|c|c|c|c|c|c|}
\hline & \multicolumn{3}{|c|}{ Homens } & \multicolumn{3}{|c|}{ Mulheres } \\
\hline & 1980 & 1990 & Variação (\%) & 1980 & 1990 & Variação (\%) \\
\hline Brasil & 6,06 & 3,58 & -41 & 4,32 & 2,41 & -44 \\
\hline \multicolumn{7}{|l|}{ Região Norte ${ }^{b}$} \\
\hline Acre & 8,49 & 5,52 & -35 & 5,36 & 3,83 & -28 \\
\hline Amapá & 6,61 & 5,25 & -20 & 5,10 & 4,41 & -13 \\
\hline Amazonas & 8,15 & 4,78 & -41 & 6,42 & 3,45 & -46 \\
\hline Pará & 7,17 & 3,90 & -46 & 5,00 & 2,78 & -44 \\
\hline Rondônia & 13,05 & 8,63 & -34 & 10,70 & 5,94 & -44 \\
\hline Roraima & 9,52 & 7,42 & -22 & 7,45 & 6,13 & -18 \\
\hline \multicolumn{7}{|l|}{ Região Nordeste } \\
\hline Alagoas & 7,96 & 4,54 & -43 & 7,12 & 3,05 & -57 \\
\hline Bahia & 5,69 & 4,15 & -27 & 4,17 & 2,81 & -33 \\
\hline Ceará & 4,13 & 2,13 & -48 & 3,23 & 1,71 & -47 \\
\hline Maranhão & 2,55 & 2,11 & -17 & 2,01 & 1,17 & -42 \\
\hline Paraíba & 6,73 & 3,65 & -46 & 4,82 & 2,66 & -45 \\
\hline Pernambuco & 6,49 & 5,18 & -20 & 4,94 & 3,65 & -26 \\
\hline Piauí & 2,72 & 2,19 & -19 & 2,22 & 1,68 & -24 \\
\hline Rio Grande do Norte & 4,77 & 2,46 & -48 & 3,65 & 1,61 & -56 \\
\hline Sergipe & 4,74 & 3,89 & -18 & 3,75 & 2,82 & -25 \\
\hline \multicolumn{7}{|l|}{ Região Centro-Oeste } \\
\hline Distrito Federal & 8,00 & 5,58 & -30 & 5,12 & 3,72 & -27 \\
\hline Goiás & 7,24 & 4,51 & -38 & 5,78 & 3,46 & -40 \\
\hline Mato Grosso & 2,93 & 3,41 & 16 & 2,20 & 2,10 & -4 \\
\hline Mato Grosso do Sul & 6,07 & 3,64 & -40 & 4,47 & 2,53 & -43 \\
\hline \multicolumn{7}{|l|}{ Região Sudeste } \\
\hline Espírito Santo & 4,31 & 2,51 & -42 & 3,01 & 2,02 & -33 \\
\hline Minas Gerais & 7,71 & 4,12 & -46 & 5,56 & 2,79 & -50 \\
\hline Rio de Janeiro & 6,85 & 4,24 & -38 & 4,45 & 2,53 & -43 \\
\hline São Paulo & 5,81 & 3,38 & -42 & 4,06 & 2,14 & -47 \\
\hline \multicolumn{7}{|l|}{ Região Sul } \\
\hline Paraná & 5,90 & 3,27 & -44 & 4,22 & 2,29 & -46 \\
\hline Rio Grande do Sul & 3,49 & 2,05 & -41 & 2,44 & 1,55 & -36 \\
\hline Santa Catarina & 3,52 & 2,07 & -41 & 2,77 & 1,61 & -42 \\
\hline
\end{tabular}

Fonte: Brasil, Ministério da Saúde, Sistema de Informações de Mortalidade, CD-ROM 1979 a 1993.

a Por 10000 habitantes.

b Para fins desta análise, o Estado de Goiás engloba o de Tocantins nos dados referentes a homens somente.

À exceção de São Paulo, estes estados (juntamente com o Pará, Minas Gerais, Espírito Santo e Mato Grosso do Sul) apresentaram, no final da década, taxas de mortalidade inferiores à média nacional $(0,48$ óbitos $/ 10000$ homens). Os demais estados, embora também tenham apresentado queda nas taxas de mortalidade por tuberculose, apresentaram variações pouco significativas e contribuíram para a permanência em níveis elevados do risco de morte por tuberculose para o sexo masculino no Brasil.

O comportamento da septicemia para a década de 80 , ao contrário das doenças infecciosas intestinais e da tuberculose, foi bastante diferenciado para os estados entre sexos e por grupos etários (estes serão discutidos mais adiante). A tabela 3 mostra que, para o Brasil, o risco de morte no sexo masculino, que já era maior que no feminino, elevou-se $(13 \%$ do início ao final da década, de 0,46 para 0,52 óbitos/10 000 homens).

Para o sexo feminino, a variação do risco de morte por septicemia foi praticamente nula (0,39 óbitos $/ 10000$ mulheres nos dois extremos da década). O comportamento adotado pelo Brasil, no entanto, foi reflexo das situações experimentadas pelos estados; observa-se que o maior risco de morte por septicemia entre os estados no início da década pertencia a Rondônia, Roraima, Rio de Janeiro e Santa Catarina, para o sexo masculino, e a Roraima e Santa Catarina, para o sexo feminino (todos com taxas acima de
0,70). Somente os estados de Rondônia, Espírito Santo, Minas Gerais, Paraná e Santa Catarina apresentaram quedas nas taxas de mortalidade para ambos os sexos. Os demais estados apresentaram aumento no risco de morte.

\section{A estrutura etária dos óbitos pelas subcausas}

O estudo do comportamento dos óbitos por doenças infecciosas e parasitárias, feito por grupos etários e por sexo, exige uma classificação dos estados, de tal forma que possa contemplar as limitações no tocante à qualidade dos registros de óbitos. Utilizou-se, como critério de classificação, o per- 
TABELA 3. Taxas padronizadas de mortalidade por doenças infecciosas intestinais, tuberculose e septicemia, conforme sexo, e percentual de variação na década, Brasil, 1980 a $1990^{a}$

\begin{tabular}{|c|c|c|c|c|c|c|c|c|c|c|c|c|c|c|c|c|c|c|}
\hline & \multicolumn{6}{|c|}{ Doenças infecciosas intestinais } & \multicolumn{6}{|c|}{ Tuberculose } & \multicolumn{6}{|c|}{ Septicemia } \\
\hline & \multicolumn{3}{|c|}{ Homens } & \multicolumn{3}{|c|}{ Mulheres } & \multicolumn{3}{|c|}{ Homens } & \multicolumn{3}{|c|}{ Mulheres } & \multicolumn{3}{|c|}{ Homens } & \multicolumn{3}{|c|}{ Mulheres } \\
\hline & 1980 & \multicolumn{2}{|r|}{$\begin{array}{c}\text { Variação } \\
(\%)\end{array}$} & 1980 & \multicolumn{2}{|c|}{$1990 \begin{array}{c}\text { Variação } \\
(\%)\end{array}$} & 1980 & \multicolumn{2}{|c|}{$1990 \begin{array}{c}\text { Variação } \\
(\%)\end{array}$} & 1980 & \multicolumn{2}{|c|}{$\begin{array}{cc} & \text { Variação } \\
1990 & (\%)\end{array}$} & 1980 & \multicolumn{2}{|c|}{$\begin{array}{cc} & \text { Variação } \\
1990 \quad(\%)\end{array}$} & 1980 & \multicolumn{2}{|c|}{$\begin{array}{cc} & \text { Variação } \\
1990 & (\%)\end{array}$} \\
\hline Brasil & 2,99 & 1,25 & -58 & 2,30 & 0,94 & -59 & 0,78 & 0,48 & -38 & 0,37 & 0,19 & -49 & 0,46 & 0,52 & 13 & 0,39 & 0,39 & 0 \\
\hline \multicolumn{19}{|l|}{ Norte } \\
\hline Acre & 3,17 & 1,28 & -60 & 2,46 & 1,34 & -45 & 1,35 & 1,05 & -22 & 0,34 & 0,58 & 70 & 0,50 & 1,22 & 144 & 0,13 & 0,62 & 377 \\
\hline Pará & 3,88 & 1,92 & -50 & 3,03 & 1,55 & -49 & 0,97 & 0,43 & -56 & 0,69 & 0,30 & -56 & 0,21 & 0,33 & 57 & 0,24 & 0,25 & 4 \\
\hline Rondônia & 4,94 & 3,19 & -35 & 3,81 & 2,94 & -23 & 1,10 & 0,74 & -33 & 0,91 & 0,61 & -33 & 0,78 & 0,76 & -2 & 0,58 & 0,54 & -7 \\
\hline Roraima & 5,04 & 1,92 & -62 & 4,11 & 1,70 & -59 & 1,12 & 1,04 & -7 & 0,00 & 0,63 & - & 0,77 & 1,07 & 39 & 1,08 & 0,80 & -26 \\
\hline Nordeste & & & & & & & & & & & & & & & & & & \\
\hline Alagoas & 5,35 & 2,34 & -56 & 4,42 & 1,79 & -59 & 0,57 & 0,40 & -30 & 0,51 & 0,23 & -55 & 0,14 & 0,57 & 307 & 0,25 & 0,45 & 80 \\
\hline Bahia & 3,22 & 1,90 & -41 & 2,54 & 1,42 & -44 & 1,04 & 0,53 & -49 & 0,65 & 0,29 & -55 & 0,28 & 0,64 & 128 & 0,25 & 0,51 & 104 \\
\hline Rio Grande do Norte & 3,35 & 1,37 & -59 & 2,70 & 1,02 & -62 & 0,55 & 0,37 & -33 & 0,28 & 0,19 & -32 & 0,33 & 0,37 & 12 & 0,23 & 0,29 & 26 \\
\hline Sergipe & 2,99 & 2,26 & -24 & 2,35 & 1,70 & -28 & 0,60 & 0,29 & -52 & 0,46 & 0,21 & -54 & 0,22 & 0,92 & 318 & 0,27 & 0,82 & 204 \\
\hline Centro-Oeste & & & & & & & & & & & & & & & & & & \\
\hline Distrito Federal & 2,34 & 1,46 & -38 & 1,62 & 1,07 & -34 & 0,83 & 0,37 & -55 & 0,49 & 0,19 & -61 & 0,16 & 0,54 & 237 & 0,18 & 0,33 & 83 \\
\hline Goiás & 1,32 & 0,39 & -70 & 1,09 & 0,30 & -72 & 0,35 & 0,09 & -74 & 0,23 & 0,04 & -83 & 0,38 & 0,45 & 18 & 0,35 & 0,38 & 8 \\
\hline Mato Grosso & 0,78 & 0,71 & -9 & 0,72 & 0,62 & -14 & 0,62 & 0,46 & -26 & 0,34 & 0,24 & -29 & 0,37 & 0,57 & 54 & 0,47 & 0,48 & 2 \\
\hline Mato Grosso do Sul & 2,73 & 1,43 & -48 & 2,32 & 1,12 & -52 & 0,83 & 0,46 & -45 & 0,40 & 0,21 & -47 & 0,63 & 0,86 & 36 & 0,62 & 0,78 & 26 \\
\hline Sudeste & & & & & & & & & & & & & & & & & & \\
\hline Espírito Santo & 2,05 & 1,08 & -47 & 1,53 & 1,03 & -33 & 0,88 & 0,41 & -53 & 0,44 & 0,19 & -57 & 0,40 & 0,35 & -12 & 0,33 & 0,31 & -6 \\
\hline Minas Gerais & 3,00 & 1,28 & -57 & 2,40 & 0,98 & -59 & 0,81 & 0,35 & -57 & 0,41 & 0,16 & -61 & 0,69 & 0,54 & -22 & 0,61 & 0,47 & -23 \\
\hline Rio de Janeiro & 3,39 & 1,35 & -60 & 2,57 & 0,98 & -62 & 1,63 & 1,22 & -25 & 0,54 & 0,36 & -33 & 0,73 & 1,06 & 45 & 0,56 & 0,79 & 41 \\
\hline São Paulo Sul & 2,84 & 0,97 & -66 & 2,20 & 0,76 & -65 & 0,73 & 0,61 & -16 & 0,29 & 0,20 & -31 & 0,60 & 0,71 & 18 & 0,51 & 0,53 & 4 \\
\hline Paraná & 2,68 & 1,53 & -43 & 2,08 & 1,20 & -42 & 0,71 & 0,29 & -59 & 0,32 & 0,12 & -62 & 0,44 & 0,40 & -9 & 0,42 & 0,35 & -17 \\
\hline
\end{tabular}

Fonte: Brasil, Ministério da Saúde, Sistema de Informações de Mortalidade, CD-ROM 1979 a 1993.

a Por 10000 habitantes.

b Para fins desta análise, o Estado de Goiás engloba o de Tocantins.

centual de causas mal definidas registrado para os estados. Desta forma, as análises dos resultados obtidos são feitas dentro do conhecimento dos níveis de sub-registro de óbitos e do percentual de causas mal definidas (5), na tentativa de minimizar erros e identificar blocos de estados com comportamentos padrões similares. Percebe-se que, ademais desses problemas, particularmente para os estados do Norte e do Nordeste, os óbitos registrados também apresentam problemas de consistência, sugeridos pela provável atração digital de algumas idades, ou seja, por erros na declaração das idades nos registros de óbito. Desta forma, são identificados quatro blocos de estados, distribuídos conforme o percentual de causas mal definidas (0-10\%; 10-20\%; $20-30 \%$; 30\% ou mais) (quadro 1).

Em praticamente todas as unidades da federação, o risco de morte por doenças infecciosas intestinais foi maior para o sexo masculino que para o sexo feminino, independente do ano estudado e da classificação adotada. Observou-se que o risco de morte concentrou-se predominantemente na população de menores de 1 ano e em menor grau na população de 70 anos e mais. A estreita relação entre a variação das taxas de mortalidade infantil por doenças infecciosas intestinais e a variação da taxa geral de mortali- dade por esta causa revelou que o coeficiente de correlação foi de 0,94 para os homens e de 0,81 para as mulheres.

O padrão etário de modo geral mostrou comportamento similar tanto para os homens como para as mulheres em 1980 e 1990, independente da classificação adotada. No entanto, os níveis dos menores de 1 ano em 1990 foram muito menos elevados que os de 1980. A semelhança entre os padrões encontrados para os diferentes agrupamentos leva à sugestão de que o padrão de mortalidade das doenças infecciosas intestinais não sofreu variação, mesmo levando em conta os distintos graus na qualidade dos dados dos estados. Ou seja, especula- 
QUADRO 1. Distribuição dos estados conforme percentual de óbitos por causas mal definidas, Brasil, 1990

\begin{tabular}{|c|c|c|}
\hline $\begin{array}{l}\text { Óbitos por causas } \\
\text { mal definidas (\%) }\end{array}$ & Homens & Mulheres \\
\hline $0-10$ & $\begin{array}{l}\text { Distrito Federal, Rio Grande do Sul, } \\
\text { Rio de Janeiro, Roraima, São Paulo }\end{array}$ & $\begin{array}{l}\text { Amapá, Distrito Federal, Rio Grande } \\
\text { do Sul, Rio de Janeiro, Roraima, } \\
\text { São Paulo }\end{array}$ \\
\hline $10-20$ & $\begin{array}{l}\text { Espírito Santo, Mato Grosso, Mato } \\
\text { Grosso do Sul, Minas Gerais, } \\
\text { Paraná, Santa Catarina }\end{array}$ & $\begin{array}{l}\text { Mato Grosso, Mato Grosso do Sul, } \\
\text { Minas Gerais, Paraná, Rondônia, } \\
\text { Santa Catarina }\end{array}$ \\
\hline $20-30$ & Goiás, Pará, Rondônia & Espírito Santo, Goiás \\
\hline$>30$ & $\begin{array}{l}\text { Acre, Alagoas, Amazonas, Amapá, } \\
\text { Bahia, Ceará, Maranhão, Paraíba, } \\
\text { Pernambuco, Piauí, Rio Grande do } \\
\text { Norte, Sergipe }\end{array}$ & $\begin{array}{l}\text { Acre, Alagoas, Amazonas, Bahia, } \\
\text { Ceará, Maranhão, Pará, Paraíba, } \\
\text { Pernambuco, Piauí, Rio Grande do } \\
\text { Norte, Sergipe }\end{array}$ \\
\hline
\end{tabular}

Fonte: Brasil, Ministério da Saúde, Sistema de Informações sobre Mortalidade, CD-ROM 1979 a 1993.

se que o padrão etário dos óbitos registrados por doenças infecciosas intestinais não é diferente dos óbitos não registrados.

Ao observar o comportamento das taxas de mortalidade por tuberculose ao longo da década de 1980, verificouse que a população do sexo masculino apresentou, de modo geral, um maior risco de morte por tuberculose, sendo que esse risco elevou-se na população em idade ativa a partir dos 35 anos, trazendo assim, junto com as causas externas e com as doenças crônicodegenerativas, um enorme prejuízo à economia brasileira. Observa-se que as taxas de mortalidade na população adulta elevaram-se de forma mais acentuada nos estados do Norte e Nordeste, regiões essas com um elevado sub-registro de óbitos. As tendências discrepantes apresentadas nessas regiões nas idades adultas, revelando um padrão não homogêneo, podem ser consequiência das deficiências já mencionadas na qualidade dos dados, adicionadas a problemas de consistência nas idades e de diagnósticos incorretos. Quanto às regiões Sul, Sudeste e Centro-Oeste, o comportamento foi mais homogêneo nos distintos grupos.

Excetuando-se os estados do Amapá, Rondônia, Mato Grosso e Acre, o padrão etário da mortalidade por tuberculose foi semelhante independente- mente da classificação dos estados, sugerindo uma conclusão semelhante à feita para as doenças infecciosas intestinais.

Em praticamente todos os estados houve um aumento no risco de morte por septicemia nos grupos mais velhos. Para ambos os sexos ficaram evidentes, nos estudos de óbitos por causa e grupos etários, os problemas de consistência dos dados de Roraima, Amapá, Rondônia e Acre, independentemente do total de óbitos por causas mal definidas.

Observou-se uma diminuição nas taxas de mortalidade por septicemia para o grupo de menores de 1 ano, com aumento de risco de morte que se deu de forma mais suave para a população adulta, exacerbando-se na população mais velha. $\mathrm{O}$ bloco dos estados com mais de $30 \%$ de óbitos por causas mal definidas diferiu dos demais blocos por apresentar, ao longo da década, um aumento no risco de morte por septicemia para a população de menores de 1 ano. Um dos fatores que poderia justificar esse aumento seria uma melhoria na capacidade diagnóstica e no preenchimento dos atestados de óbito para essa população. Os estados do Norte, com exceção de Amazonas e Pará, caracterizaram-se por apresentar fortes evidências de subregistro de óbitos e indícios de imprecisão no registro de dados.

\section{CONCLUSÕES}

O grande saldo verificado na década de 1980 foi a diminuição significativa dos níveis de mortalidade por doenças infecciosas e parasitárias em todo o Brasil e em praticamente todas as faixas etárias, em particular na faixa das crianças com menos de 1 ano. Contudo, a situação ainda não é ideal. As taxas de mortalidade brasileira por doenças infecciosas ainda são elevadas para os padrões mundiais na atualidade, especialmente para o grupo de menores de 1 ano e em particular para os estados menos desenvolvidos, em sua quase totalidade pertencentes às regiões Norte e Nordeste. Estes níveis ainda elevados orientam para a prioridade de atuação junto a esses grupos etários.

Ainda são perdidos muitos anos potenciais de vida por causas que seriam evitáveis com ações básicas de prevenção e atenção primária à saúde, de baixo custo. A cobertura da assistência médica à população, em particular nas localidades carentes, ainda é baixa, o que resulta na elevada prevalência da morbidade e da mortalidade. É necessário insistir para que os registros de eventos vitais sejam corretamente preenchidos; além disso, os órgãos competentes devem exercer um controle mais rigoroso da qualidade destes registros. Destaca-se a necessidade da criação de sistemas de vigilância epidemiológica e, nacionalmente, da implantação dos serviços de verificação de óbitos, se não em todas as localidades, pelo menos em localidades de referência.

A construção de indicadores de mortalidade para países em desenvolvimento ainda merece ser alvo de críticas e estudos, em particular no Brasil. Dentro da literatura mundial, as estatísticas de mortalidade de países em desenvolvimento têm resultado em exaustivas discussões quanto ao seu poder preditivo e quanto à capacidade de identificar níveis e tendências de mortalidade. Apesar dos dados provenientes dos registros de óbitos no Brasil apresentarem problemas de consistência, foi possível desenhar um quadro geral da mortalidade, sempre com interpretações cautelosas. 


\section{REFERÊNCIAS}

1. Omran AR. The epidemiologic transition: a theory of the epidemiology of population change. Milbank Mem Found Q 1971;49(4): 509-538.

2. Frenk J, Frejka T, Bobadilha JL, Stern C, Lozano R, José M. La transición epidemiológica en América Latina. Bol Oficina Sanit Panam 1991;111(6):485-496.

3. Barreto ML, Carmo EA. Mudanças nos padrões de morbimortalidade. Em: Monteiro CA. Velhos e novos males da saúde no Brasil: a evolução do país e de suas doenças. São Paulo: Editora Hucitec NUPENS/Universidade de São Paulo; 1995. p. 17-30.

4. Paes NA. Model life representation for Brazilian mortality [tese de doutorado]. London: London School of Hygiene and Tropical Medicine, University of London: 1993.

5. Paes NA. O poder explicativo dos registros de óbitos para estimar taxas de mortalidade no Brasil [relatório técnico]. Brasília, DF: CNPq; 1997
6. Chackiel J. La investigación sobre causas de morte en la América Latina. Notas de Poblacion 1987;44:9-30.

7. Preston SH. Use of direct and indirect techniques for estimating the completeness of death registration systems: data bases for mortality measurement (Bangkok, 20-23). New York: United Nations; 1984.

8. Brasil, Ministério da Saúde. Sistema de Informação sobre Mortalidade, 1979-1993 [base da dados em CD-ROM]. Brasília, DF: CENEPI; 1995.

9. Fundação Instituto Brasileiro de Geografia e Estatística. IX Recenseamento geral do Brasil: 1980, censo demográfico. Rio de Janeiro: IBGE; 1983.

10. Fundação Instituto Brasileiro de Geografia e Estatística. X Recenseamento geral do Brasil: 1990, censo demográfico. Rio de Janeiro: IBGE; 1991.

11. Juliano Y, Novo NF, Goihman S, Paiva ER, Leser W. Coeficiente padronizado de anos de vida perdidos, de Haenszel: comparação com o coeficiente padronizado de mortalidade geral, quanto à utilização como indicador do nível de saúde da população. Rev Saude Publica 1987;21(2):108-122.

12. Waldman EA, Silva LJ, Monteiro CA. Trajetória das doenças infecciosas: da eliminação da poliomielite à reintrodução da cólera. Em: Monteiro CA. Velhos e novos males da saúde no Brasil: A evolução do país e de suas doenças. São Paulo: Editora Hucitec NUPENS/Universidade de São Paulo; 1995. p. 17-30.

Manuscrito recebido em 31 de janeiro de 1998. Aceito em versão revisada em 21 de dezembro de 1998.

ABSTRACT Brazil has been undergoing a period of epidemiological and demographic transition, which has included an improvement in the quality of death certificate registrations and major changes in the patterns of mortality from infectious and parasitic diseases.

Infectious and parasitic diseases in Brazil: a decade of transition
This article outlines the changes in the mortality patterns that were observed in the country and in its states during the decade of the 1980s. We used data from the Ministry of Health Mortality Information System, classified according to the International Classification of Diseases, 9th Revision. Our analysis showed important changes in mortality patterns in Brazil. Mortality from infectious diseases decreased by $41 \%$ among men and by $44 \%$ among women. While these types of changes were especially noticeable in the states of the North and Northeast, these states still have the highest mortality rates in the country. The changes particularly affected the extreme limits of the age continuum, most especially children under 1 year of age. Within the group of infectious and parasitic diseases, we also assessed the mortality due to intestinal infectious diseases, tuberculosis, and septicemia. We found that in the 1980s there was a major decrease in the rates of mortality due to intestinal infectious diseases and to tuberculosis. However, there was an increase in the risk of death from septicemia during the decade. In conclusion, we find that the rate of mortality caused by infectious and parasitic diseases remains high in Brazil. Therefore, Brazilian health authorities still need to give priority attention to this cause of death. 